\title{
Impactos socioeconómicos del cambio climático en América Latina y el Caribe:
} 2020-2045*

Jesús J. Rodríguez De Luque**

Carlos E. González Rodríguez ${ }^{* * *}$

Sharon Gourdji****

Daniel Mason-D'Croz ${ }^{* * * * *}$

Diego Obando-Bonilla******

Jeison Mesa-Diez ${ }^{* * * * * * *}$

Steven D. Prager ${ }^{* * * * * * * *}$

doi:10.III44/Javeriana.cdri3-78.iscc

Recibido: 2016-06-03 Aprobado: 2016-09-27 Disponible en línea: 2016-12-20

Cómo citar este artículo: Rodríguez De Luque, J. J., Gonzalez, C., Gourdji, S., Mason-D’Croz, D., ObandoBonilla, D., Mesa-Diez, J. y Prager, S. D. (2016). Impactos socioeconómicos del cambio climático en América Latina y el Caribe: 2020-2045. Cuadernos de Desarrollo Rural, I3(78), II-34. https://doi.org/10.III44/Javeriana.cdriz-78.iscc

\footnotetext{
* Artículo de investigación. La investigación que dio origen a este artículo contó con al colaboración de los programas del CGIAR en Cambio Climático, Agricultura y Seguridad Alimentaria (CCAFS), y Políticas, Instituciones y Mercados (PIM). Así como también de Global Futures \&o Strategic Foresight y Climate Change Vulnerability in the Agricultural Sector en LAC, CIAT e Ifpri.

** Magister en Economía. Anterior Asociado de Investigación en el Centro Internacional de Agricultura Tropical (CIAT). Correo electrónico: rodriguezjesusjose@hotmail.com. Orcid: http://orcid. org/0000-0002-8179-0109

*** Magister en Economía. Asociado de Investigación en el Centro Internacional de Agricultura Tropical (CIAT). Correo electrónico: c.e.gonzalez@cgiar.org. Orcid: http://orcid.org/0000-0002-4167-0209

**** Doctora en Ingeniería Ambiental. Ingeniera Ambiental en el National Institute of Standards \& Technology (NIST). Correo electrónico: sharon.gourdji@nist.gov. Orcid: http://orcid. org/0000-0002-0309-9187

***** Magister en Economía Internacional. Científico en el International Food Policy Research Institute (Ifpri). Correo electrónico: d.mason-dcroz@cgiar.org. Orcid: http://orcid.org/0000-0003-0673-230I ******* Estadístico. Asistente de investigación en el Centro Internacional de Agricultura Tropical (CIAT). Correo electrónico: j.mesa@cgiar.org. Orcid: http://orcid.org/0000-0002-8262-740X ******** Doctor en Geografía. Científico Sénior en el Centro Internacional de Agricultura Tropical (CIAT). Correo electrónico: s.prager@cgiar.org. Orcid: http://orcid.org/0000-000I-9830-7008
} 


\title{
Resumen
}

En América Latina y el Caribe (ALC), el arroz, el trigo, el maíz, el frijol y la soya juegan un papel importante debido a sus aportes a la economía y a la seguridad alimentaria. Dada la existencia de evidencia que señala que a nivel mundial se presentarían cambios en el clima en el mediano plazo, en el presente texto se evalúan los impactos socioeconómicos que habría a niveles de ALC si no fueran implementadas medidas de adaptación. Con este fin, se realiza una integración entre modelos climáticos, de cultivos y económicos. Los resultados muestran que los crecimientos de las producciones de maíz y frijol caerían de manera importante en Nicaragua, El Salvador, Guatemala, Honduras, Colombia, Venezuela y Brasil, y los del arroz y trigo presentarían importantes disminuciones en Brasil, Argentina y Uruguay. Finalmente, se colige que el cambio climático tiene la capacidad de frenar parte de los avances en materia de seguridad alimentaria en la región, debido a sus efectos negativos sobre la disponibilidad de alimentos.

\section{Palabras clave:}

cambio climático; agricultura; seguridad alimentaria; América Latina; Caribe; economía

\section{Socio-Economic Impacts of Climate Change in Latin America and the Caribbean: 2020-2045}

\begin{abstract}
Rice, wheat, corn, beans, and soybeans play an important role in Latin America and the Caribbean (LAC) due to their contributions to economy and food security. Given that there is evidence that there would be mid-term climate changes on a global scale; this article assesses the socio-economic impacts that would take place at LAC levels if adaptation measures were not implemented. To this end, we carried out an integration between climate, crop, and economic models. Results show that the growth of corn and beans production would fall significantly in Nicaragua, El Salvador, Guatemala, Honduras, Colombia, Venezuela and Brazil, and rice and wheat would decline significantly in Brazil, Argentina and Uruguay. Finally, we found that climate change has the capacity to partially curb the progress in food security in the region, due to its negative effects on food availability.
\end{abstract}

Keywords:

climate change; agriculture; food security; Latin America; Caribbean; economy 


\section{Introducción}

Los impactos que tendría el cambio climático en el mediano plazo sobre los sistemas de producción agrícola conforman un foco de atención cuyo abordaje tiende a darse con creciente interés en América Latina y el Caribe. Esto se debe a la existencia de evidencia la cual señala que en las próximas décadas se presentarían cambios en los patrones de lluvias y las temperaturas, al igual que incrementos notables en la variabilidad climática a nivel mundial (IPCC Working Group, 2013). Dichos cambios ambientales tienen el potencial de afectar la productividad agrícola y la seguridad alimentaria. En ese sentido, los involucrados del sector agrícola están interesados en comprender tales impactos para poder así diseñar e implementar programas y políticas públicas que permitan enfrentarlo (Meadu, Coche, Vermeulen y Engelund Friss, 2015).

Los cambios en el clima pueden afectar los rendimientos y la producción agrícola mediante diversos mecanismos. Entre los más importantes se encuentran los incrementos en la frecuencia de estrés por aumento de temperatura en la etapa reproductiva de las plantas (Gourdji, Sibley y Lobell, 2013); los incrementos en estrés por agua debido a disminuciones o incrementos de las lluvias (IPCC Working Group, 2013), y las reducciones en el periodo de crecimiento debido a las elevadas temperaturas (Vaghefi, Nasir Shamsudin, Radam y Rahim, 2013). Estudios recientes han encontrado que los cambios en las temperaturas y en los patrones de lluvias podrían ocasionar que lugares actualmente aptos para la siembra de algunos cultivos dejen de serlo en las próximas décadas (Jarvis, Ramírez, Herrera y Navarro, 20I2). Estos resultados sugieren que el cambio climático puede ocasionar alteraciones importantes en la frontera agrícola y, por tanto, en los sistemas socioeconómicos.

En cuanto al impacto potencial que tendría el cambio climático en los cultivos analizados en este estudio, investigaciones recientes indican que para la década de 2030 el 3r \%, el i6 \% y el ir \% de la producción mundial de maíz, arroz y trigo, respectivamente, pueden verse afectadas por al menos cinco días de altas temperaturas en la etapa reproductiva en un año típico. Dado que las plantas son especialmente sensibles a las altas temperaturas durante esta etapa, lo anterior puede afectar de manera importante los rendimientos, si las medidas de adaptación no son tomadas adecuadamente (Gourdji et al., 20I3). Sin embargo, los impactos de las altas temperaturas sobre los rendimientos dependen del cultivo y del tipo de sistema afectado. Para el caso del maíz, resultados encontrados por Lobell, Banziger, Magorokosho y Vivek (20II) señalan que sus rendimientos en condiciones de secano óptimas y de sequía pueden disminuir en porcentajes de I \% y r.7 \%, respectivamente, por cada r adicional con que se eleve por encima de los durante el día. 
Por otra parte, evidencia encontrada en el marco del Agricultural Model Intercomparison and Improvement Project (AgMIP) indica que los impactos que el cambio climático tendría sobre los rendimientos del trigo, cereales secundarios, arroz y semillas oleaginosas, pueden también afectar sus producciones y precios, y de esta manera su disponibilidad y accesibilidad. En otras palabras, se encontró que los cambios en el clima pueden generar reducciones del iI \% en sus rendimientos e incrementos de un $20 \%$ en sus precios, en comparación a un escenario sin cambios en el clima, si no son tomadas medidas de adaptación (Nelson et al., 20I4)

En este orden de ideas, el objetivo general de este artículo es evaluar los impactos socioeconómicos que el cambio climático tendría a nivel de país en ALC entre 2020 y 2045. Con este propósito, se realiza una integración entre modelos climáticos, de cultivos y económicos. En otras palabras, se utilizan simulaciones de los rendimientos del frijol, el arroz, el maíz, la soya y el trigo que fueron realizadas con el software Decision Support System for Agrotechnology Transfer (Dssat) (Jones et al., 2003) bajo distintos modelos climáticos. Luego, esta información fue incorporada al International Model for Policy Analysis of Agricultural Commodities and Trade (Impact) (Robinson et al., 2015) para construir escenarios factibles de la evolución de los mercados agrícolas, así como la seguridad alimentaria de los países latinoamericanos en el contexto de los cambios en los patrones de lluvias y las temperaturas. Los objetivos específicos son: a) evaluar los efectos que el cambio climático tendría sobre los mercados del frijol, el arroz, el trigo, el maíz y la soya, si no son tomadas medidas de adaptación, y b) estudiar las consecuencias del cambio climático sobre la seguridad alimentaria de los países latinoamericanos.

En sus dos objetivos, este artículo se diferencia de los antecedentes disponibles al evaluar las consecuencias del cambio climático a nivel de país en América Latina y el Caribe. Su contribución principal radica en aportar información útil para distintos debates que están vigentes en la región, relacionados con las medidas que son necesarias para enfrentar los cambios en el clima en el mediano plazo, así como los costos potenciales que tendría la ausencia de medidas de adaptación.

El resto del presente artículo se organiza de la siguiente manera: luego de esta sección introductoria, en la segunda se explican los materiales y métodos que son empleados para responder las preguntas de investigación. A continuación se presentan los resultados de las simulaciones que competen a los impactos del cambio climático, a nivel de país, en los mercados agrícolas de los cultivos analizados al igual que a la seguridad alimentaria de los países latinoamericanos. En la siguiente sección se discutirán los resultados. Por último, en la quinta se presentan las conclusiones. 


\section{Materiales y métodos}

Impact es un modelo de equilibrio parcial global en el que se representa la producción, el comercio internacional, la demanda y los precios de productos agrícolas (39 cultivos, 6 productos de ganadería y i7 productos procesados). En su versión actual, el modelo incluye una desagregación geográfica de 320 unidades productoras de alimentos (FPU, por sus siglas en inglés), la cual se realiza con base en la relación de 159 países y 154 cuencas hidrográficas. Impact es un modelo con diseño modular, por lo que es posible integrarlo con otros, como por ejemplo diversos modelos climáticos, General Circulation Models (GCMs), de cultivos (Dssat), de seguridad alimentaria y de uso de la tierra, entre otros. En Robinson et al. (2015) se presenta una descripción detallada del modelo, sus supuestos, ecuaciones, regiones geográficas, cultivos, productos de ganadería y módulos incorporados, entre otros.

Las principales fortalezas de Impact frente a otros modelos son la desagregación geográfica y de productos agrícolas, ya que representa más países y productos directamente. Además, a este nivel de desagregación, el modelo permite incluir el conocimiento experto de cultivos de instituciones de investigación agrícola. Es por ello que hasta ahora todos los estudios del AgMIP han usado las tazas de crecimiento de la productividad de Impact. El modelo también tiene una larga historia de uso en temas de seguridad alimentaria; cambios en el sistema agrícola global, y cuestiones alusivas al cambio climático. Gracias a su diseño modular, es posible integrarlo con otros modelos cuando es necesario. Dentro de Impact este conjunto de circunstancias permite tener un módulo hidrológico y de manejo de recursos de agua; algo que otros modelos no tienen. Fuera de Impact, su diseño ha permitido integrarlo con modelos de uso del suelo, así como con modelos de equilibrio general y análisis de biodiversidad, entre otros.

Sin embargo, otros también tienen puntos a su favor. En ese sentido, Impact es un modelo de equilibrio parcial; una herramienta que permite una desagregación amplia en el sector agrícola, pero que requiere sacrificar la conexión directa a otros sectores de la economía, como se puede hacer en un modelo de equilibrio general como AIM (Fujimori, Masui y Matsuoka, 20I2), Envisage (Van Der Mensbrugghe, 20I0) y FARM (Darwin, 1998). Impact tiene un modelo de uso de suelos; sin embargo, este módulo es bastante simple, y no considera todos los usos potenciales de la tierra, ya que solo se enfoca en su uso con fines de siembra de cultivos. Globiom (Havlík et al., 20I4) y MAgPIE (PIK Landuse Group, 20II), por otra parte, tienen módulos de uso del suelo mucho más detallados, y consideran distintos usos de la tierra. 
No todos estos prototipos usan Dssat como su modelo de simulación de cultivo. Esto tiene sus ventajas y sus desventajas. Por ejemplo, Globiom usa EPIC. Esto les permite simular directamente más cultivos en su modelo que lo que se puede hacer en Impact actualmente. Sin embargo, ellos simulan el cambio climático asumiendo que va a haber fertilización de $\mathrm{CO}_{2}$, algo que puede hacer que los resultados que consideran en torno al impacto del cambio climático sean optimistas (Havlík et al., 20r4)

En cuanto a los insumos utilizados, aquí se emplean tres bases de datos, entre las que se incluyen los rendimientos simulados de los cultivos; las proyecciones de los crecimientos de la productividad agrícola, y diversas proyecciones alusivas al incremento de la población y el crecimiento económico al nivel del país. En conjunto, estos insumos son usados para plantear escenarios factibles para la evolución de los mercados agrícolas y la seguridad alimentaria en ALC, dados los cambios en la productividad agrícola; los crecimientos económicos y poblacionales, y el cambio climático.

Ante todo, los rendimientos utilizados en este estudio provienen de dos fuentes de información. Primero, para el caso de los cultivos de arroz, trigo, maíz, soya y frijol, se emplean los rendimientos de estos cultivos simulados en nueve escenarios de cambio climático y en un escenario sin cambio climático (ver tabla I). Dichos resultados fueron desarrollados en el marco del proyecto Climate change vulnerability in the agricultural sector in Latin America and the Caribbean, el cual fue desarrollado en el Centro Internacional de Agricultura Tropical. En el trabajo de Gourdji et al. (2016) se presenta una explicación detallada del proceso de modelación de los cultivos aquí analizados. En segundo lugar, para los demás cultivos presentes en el modelo Impact, este estudio se beneficia de la última versión de los impactos del cambio climático presentes en dicho modelo; resultados que fueron desarrollados en el marco del proyecto Global Futures E Strategic Foresight. Así pues, en el estudio de Robinson et al. (2015) se presenta una descripción de la metodología desarrollada en este último caso.

Para la simulación de los rendimientos de los cultivos analizados en este estudio se usaron, además de los datos de clima, información detallada de suelos, variedad de cultivo, fechas de siembra, densidad, aplicaciones de fertilizante, tecnologías de riego o secano, entre otros. Con este fin fueron utilizadas bases de datos espaciales globales a escala $0.5^{\circ}$ de resolución que permitían identificar la presencia de cultivo, para lo que se utilizó la base del Spatial Production Allocation Model (SPAM) 2005 v2.0 (You et al., 20I4). La información de suelos usada principalmente provino del Harmonized World Soil Database de FAO et al. (2012). Además, las fechas de siembra fueron determinadas con base en los trabajos de Portman, Siebert y Doll (2010). Por 
último, es importante destacar que en la simulación de los rendimientos se asumió que no se implementaban medidas de adaptación que se ajustaran a los impactos del cambio climático; por ello, las áreas de cultivo son fijas en los periodos pasado (19751998) y futuro (2020-2045) (Gourdji et al., 2016).

TABLA 1. Datos climáticos usados en las simulaciones de los rendimientos desarrollados en Dssat

\begin{tabular}{llll}
\hline Escenario & Cultivos & Región & Datos climáticos \\
\hline $\begin{array}{l}\text { Cambio } \\
\text { climático (CC) }\end{array}$ & $\begin{array}{l}\text { Frijol, arroz, } \\
\text { maíz, trigo, } \\
\text { y soya }\end{array}$ & ALC & $\begin{array}{l}\text { Bcc-Csmi, Bnu_Esm, Cccma_Canesm2, } \\
\text { Gfld_Esm2g, Inm-Cm4, Ips- } \\
\text { Cm5a-Lr, Miroc-Miroc5, Mpi- } \\
\text { Esm-Mr, y Ncc-Noresmi-M }\end{array}$ \\
\hline Cambio climático & $\begin{array}{l}\text { Otros cultivos } \\
\text { en Impact }\end{array}$ & $\begin{array}{l}\text { ALC y resto } \\
\text { del mundo }\end{array}$ & $\begin{array}{l}\text { Promedio del impacto del cambio } \\
\text { climático bajo los modelos Gfdl_Esm2, } \\
\text { Hadgem2_Es, Ipsl_Cm5a_Lr, y ALC y } \\
\text { resto del mundo Miroc_Esm_Chem }\end{array}$ \\
\hline \multirow{2}{*}{$\begin{array}{l}\text { Ausencia de } \\
\text { cambio climático }\end{array}$} & $\begin{array}{l}\text { Frijol, arroz, } \\
\text { maíz, trigo, } \\
\text { y soya }\end{array}$ & ALC & Watch Forcing Dataset (WFD) \\
\hline \multirow{2}{*}{ NoCC } & $\begin{array}{l}\text { Otros cultivos } \\
\text { en Impact }\end{array}$ & & Watch Forcing Dataset (WFD \\
\hline
\end{tabular}

FuENTE: los autores

La integración de los resultados de Dssat a escala $0.5^{\circ}$ de resolución con el modelo Impact se realizó, primero, agregando los rendimientos simulados a la escala más pequeña que tiene el modelo Impact, que corresponde a las FPU. Posteriormente, y siguiendo la metodología propuesta por Robinson et al. (2015), el impacto que el cambio climático tiene sobre los rendimientos de los cultivos es calculado de acuerdo con las tasas de crecimiento anual que pueden observarse entre los periodos pasado y futuro.

Los rendimientos de los cultivos en el modelo Impact, además de verse afectados por el cambio climático, se ven influidos también por los incrementos de la productividad agrícola. En Impact se asume que el crecimiento de la productividad sigue una trayectoria establecida por las llamadas tasas de crecimiento intrínsecas (IPRs, por sus siglas en inglés). Las IPRs son supuestos acerca de cómo puede evolucionar la productividad de los cultivos en el tiempo debido a avances tecnológicos. Las IPRs fueron calculadas inicialmente con base en la metodología propuesta por Evenson, Pray y Rosegrant (1999), y han sido ajustadas a partir de consultas a expertos (Robinson et al., 2015). 
Finalmente, para complementar la definición de los escenarios aducidos, se emplean las proyecciones de los Share Socio-Economic Pathways (SSPs) desarrollados por el International Institute for Applied Systems Analysis (Iiasa) acerca del crecimiento poblacional y el Producto Interno Bruto (PIB). Las proyecciones de los SSPs buscan describir la posible evolución de ciertos factores socioeconómicos (demográficos, económicos, políticos, sociales, culturales, institucionales y tecnológicos) que pueden hacer más fácil o difícil la toma de medidas de mitigación y/o adaptación al cambio climático (O’Neill et al., 20I4). Específicamente, aquí se utilizan las proyecciones del escenario $\mathrm{SSP}_{2}$. Este escenario describe un mundo en donde la mayoría de las tendencias que están actualmente relacionadas con los patrones de consumo de energía y desarrollo económico, continúan en el futuro, pero algunos progresos son realizados para reducir el consumo de combustibles fósiles (O’Neill et al., 20I4).

En las secciones siguientes se presentan las evaluaciones realizadas. Todos los datos presentados a partir de ahora son resultados del modelo Impact, o bien corresponden a cálculos realizados a partir de ellos. Entre las variables analizadas del modelo Impact se encuentran los rendimientos, el área cultivada, la producción, la demanda, las exportaciones netas, los precios mundiales, y el porcentaje de la población en riesgo de padecer hambre. En la tabla 2 se presenta una explicación de cada una de las variables analizadas y de sus ecuaciones.

TABLa 2. Descripción de las variables analizadas del modelo Impact

Los rendimientos de los cultivos (Yield) corresponden a una función de los rendimientos en el año base (YieldInt); un multiplicador de crecimiento exógeno (YieldInt2), los precios propios, los precios de los insumos, la disponibilidad de agua y una tendencia exógena. Los cambios en los rendimientos se deben a cuatro factores: las IPRs; la respuesta endógena de los rendimientos a cambios en los precios de los insumos y el precio propio en el corto plazo; la respuesta de los rendimientos ante cambios en el clima, calculados en DSSAT y en el sub-módulo de agua de Impact, y el Rendimientos estrés por agua, que es calculado también en el módulo de agua de Impact (WatShk). En este estudio el módulo de agua de Impact no fue usado.

$$
\begin{aligned}
\text { Yield }_{j, f p u, \text { Ind }} & =\text { Yieldlnt }_{j, f p u, \text { Ind }} \times \text { Yieldlnt2 }_{j, f p u, \text { Ind }} \times \text { WatShk }_{j, f p u, \text { Ind }} \\
& \times \text { CliShk }_{j, f p u, \text { Ind }} \times\left(\frac{P N E T_{j, c t y}}{P N E T 0_{j, c t y}}\right)^{Y \varepsilon} \times P F^{F \varepsilon}
\end{aligned}
$$


La demanda por área corresponde a una función de área de cultivo en el año base (AreaInt); a un multiplicador exógeno del crecimiento del área cultivada (AreaInt2); a la elasticidad de la demanda con respecto al precio sombra de la tierra ; al producto marginal del ingreso en el año base (MRPo), y a la elasticidad de la demanda Área cultivada de área con respecto al producto marginal del ingreso.

$$
\begin{aligned}
& \text { Area }_{j, f p u, l n d}=\text { Arealnt }_{j, f p u, l n d} \times \text { Arealnt }_{j, f p u, l n d} \times W F_{f p u, I n d}^{W F} \\
& \times\left(\frac{M R P_{j, f p u}}{M R P 0_{j, f p u}}\right)^{A \varepsilon}
\end{aligned}
$$

La producción de cultivos (QS) corresponde a una función de los rendimientos y el área cultivada:

Producción

DE CUltivos

$$
Q S_{j, \text { ty }}=\sum_{\text {fpu, Ind }}\left(\text { Area }_{j, f p u, \text { Ind }} \times \text { Yield }_{j, f p u, \text { Ind }}\right)
$$

La demanda doméstica total por un producto (QD) es representada como la suma de la demanda de comida por los hogares $(\mathrm{QH})$ (la cual es una función, entre otros factores, del PIB, la población total, y los precios); la demanda agrícola para fines intermedios (QInterm); la demanda de alimentos para alimentar el ganado (QL); la demanda intermedia para producir Demanda biocombustibles (QBF), y la demanda intermedia de otros sectores (QOth):

$$
Q D_{c, c t y}=\sum_{H}\left(Q H_{c, h, c t y}\right)+Q I n t e r m_{c, t y}+Q L_{c, t y}+Q B F_{c, t y}+Q O t h_{c, t y y}
$$

Las exportaciones netas (NT) son una función de la producción, la

EXPORTACIONES demanda doméstica (QD) y el cambio en reservas (QSt).

NETAS

$$
N T_{c, \text { CTY }}=Q S U P_{c, \text { ty }}-Q D_{c, \text { try }}-Q S T_{c, \text { ty }}
$$

Precios El precio mundial de un producto es aquel que equilibra los mercados MUNDiales domésticos e internacionales para los productos transables.

Porcentaje de Este indicador se calcula con base en resultados encontrados LA POBlación $\quad$ por Fischer, Shah, Tubiello y Velhuizen (2005) que muestran EN RIESGO DE la existencia de una relación empírica entre el porcentaje de la Padecer hambre población en desnutrición y la disponibilidad relativa de comida.

Fuente: adaptado de Robinson et al. (2015) 


\section{Resultados}

\section{Incertidumbre respecto a los impactos del cambio climático}

La evaluación de los impactos del cambio climático es un proceso que tiene distintos tipos de incertidumbre, que están asociados con los materiales y métodos empleados en las distintas fases de la modelación. Para ilustrar la variabilidad de las proyecciones realizadas, en esta subsección se presentan, en el gráfico I, las tasas de crecimientos de las producciones simuladas en Impact para cada cultivo y país analizado, bajo los nueve GCMs y un escenario en el que el cambio climático no se presenta. Una primera observación que se desprende de dicho gráfico es que hay países en donde el rango de las proyecciones es más amplio que en otros. En otras palabras, para algunos países la incertidumbre respecto a los efectos de los cambios en el clima en el crecimiento de las producciones es mayor que en otros. Por ejemplo, para el caso del frijol, la dispersión de las proyecciones es relativamente mayor en países como Venezuela, Cuba, Haití, El Salvador, Nicaragua y Belice. Lo mismo pasa con el maíz en países como Jamaica, Uruguay, Venezuela, Belice, Colombia y Costa Rica.

La varianza en los resultados de Impact refleja las diferencias que se observan en los rendimientos de los cultivos simulados en Dssat en cada uno de los nueve GCMs. Debido a la variabilidad de las proyecciones realizadas, en las siguientes subsecciones se emplea el valor mediano de los impactos del cambio climático sobre las variables y cultivos analizados. Así se busca presentar resultados conservadores respecto a estos impactos sobre los rendimientos, las áreas cultivadas, las producciones, las demandas, los precios y la seguridad alimentaria de los países analizados.

\section{Rendimiento, área cultivada, producción y demanda}

Los resultados sugieren que el cambio climático tendría, en general, un impacto negativo sobre el crecimiento de los rendimientos de los cultivos analizados. En otras palabras, se encuentra que debido a los incrementos en la frecuencia de sequías y en los periodos calurosos, los rendimientos tendrían un crecimiento mediano de $15.6 \%$ entre 2020 y 2045 , mientras que en el escenario sin cambios en el clima su crecimiento sería del $23 \%$. Esto significa que, a nivel regional, el cambio climático tendría un impacto negativo sobre el crecimiento de los rendimientos igual al 32.2 $\%$. No obstante, es importante anotar que los efectos del cambio climático serían heterogéneos entre cultivos y países, como se puede ver en el gráfico 2 y en el gráfico 3. 


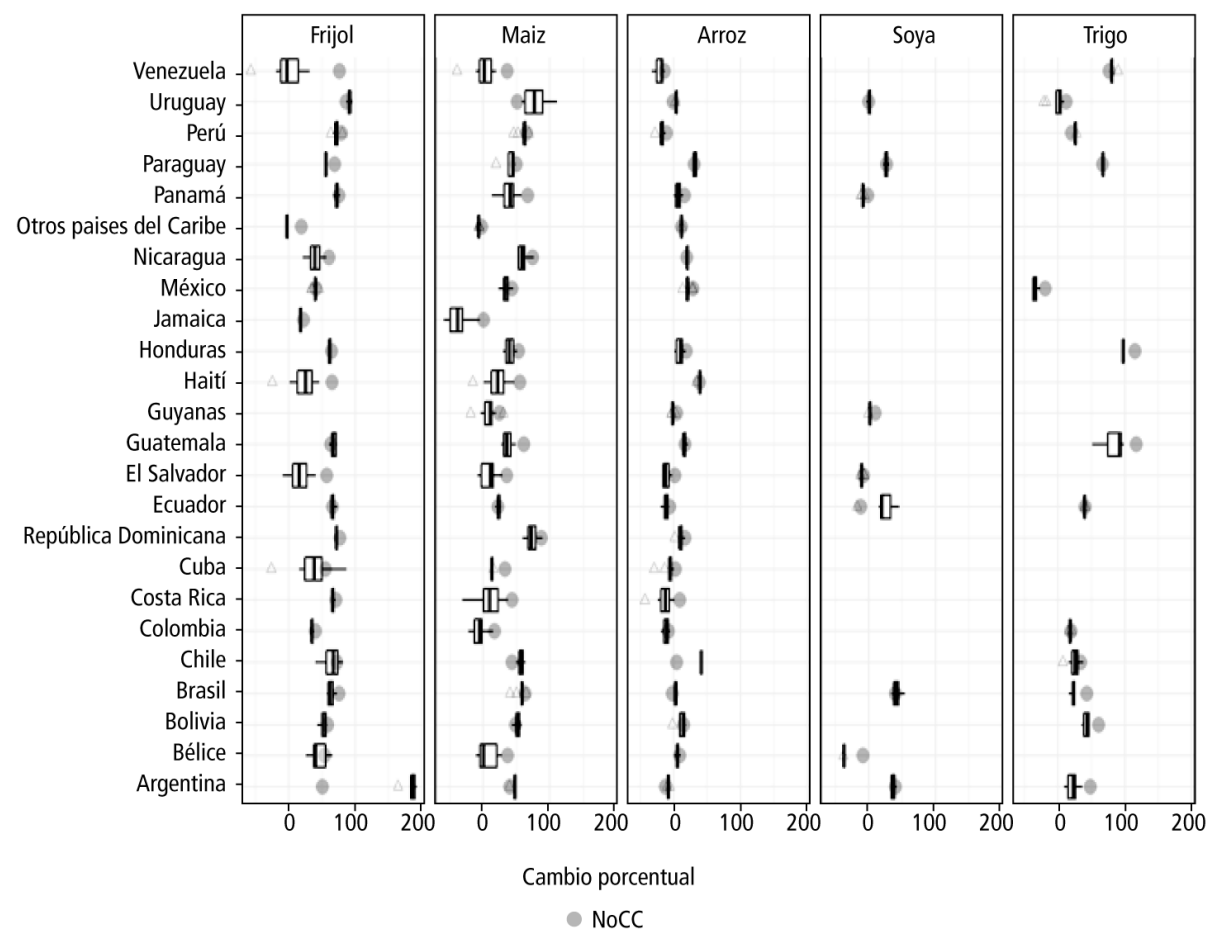

GRÁfICo l. Diagrama de caja de los crecimientos de la producción por países y cultivos para los escenarios con y sin cambio climático

Fuente: cálculos propios

Nota: el cambio porcentual se calcula con base en los valores en 2020 y 2045 de la producción para cada escenario de cambios climático y el escenario sin cambio climático, $\left(\frac{X_{2045}^{\mathrm{cc}}-X_{2020}^{\mathrm{cc}}}{X_{2020}^{\mathrm{cc}}}\right)$ y $\Delta \% X_{2045-2020}^{\mathrm{cc}}=\left(\frac{X_{2045}^{\mathrm{cc}}-X_{2020}^{\mathrm{cc}}}{X_{2020}^{\mathrm{Cc}}}\right)$

Dichos cambios en los rendimientos tendrían efectos sobre la rentabilidad de los cultivos, por lo que los agricultores estarían incentivados a modificar la asignación de tierra entre ellos. Los resultados indican que el crecimiento del área cultivada en el escenario mediano equivaldría al $8.82 \%$, y en ausencia de cambios en el clima sería de $13 \%$. Los mencionados impactos del cambio climático sobre el crecimiento de los rendimientos y las áreas cultivadas generarían una caída mediana en el crecimiento de las producciones de los cultivos analizados del $34.3 \%$, respecto al escenario que no involucra cambios en el clima. Por otra parte, el crecimiento de la demanda doméstica de los cultivos analizados no se vería muy afectado, pues esta variable presentaría una caída relativa que correspondería al $2.67 \%$. 
Por otra parte, la evidencia encontrada muestra que el cambio climático tendría efectos específicos por cultivos y países, como puede verse en el gráfico 3. Para el caso del maíz y el frijol, se nota que el crecimiento de sus rendimientos se vería afectado negativamente en países como Venezuela, Nicaragua, Jamaica, Haití, El Salvador, Cuba, Costa Rica, Belice y Brasil, mientras que en Uruguay y Chile el crecimiento de los rendimientos del maíz, y en Argentina los del frijol se verían afectados positivamente. Para el caso de la soya, la evidencia encontrada indica que el crecimiento de sus rendimientos se vería afectado negativamente en países como Uruguay, Argentina y Ecuador, y en el resto de países productores los impactos del cambio climático serían positivos o neutrales. El cultivo de trigo, por su parte, experimentaría reducciones en el crecimiento de sus rendimientos, especialmente en los grandes productores regionales como México, Argentina, Brasil y Uruguay. Finalmente, el crecimiento de los rendimientos del arroz se vería afectado negativamente en muchos países de Centro América, el Caribe y México, mientras que en Colombia, Venezuela, Brasil, Chile y Uruguay, experimentaría impactos positivos.

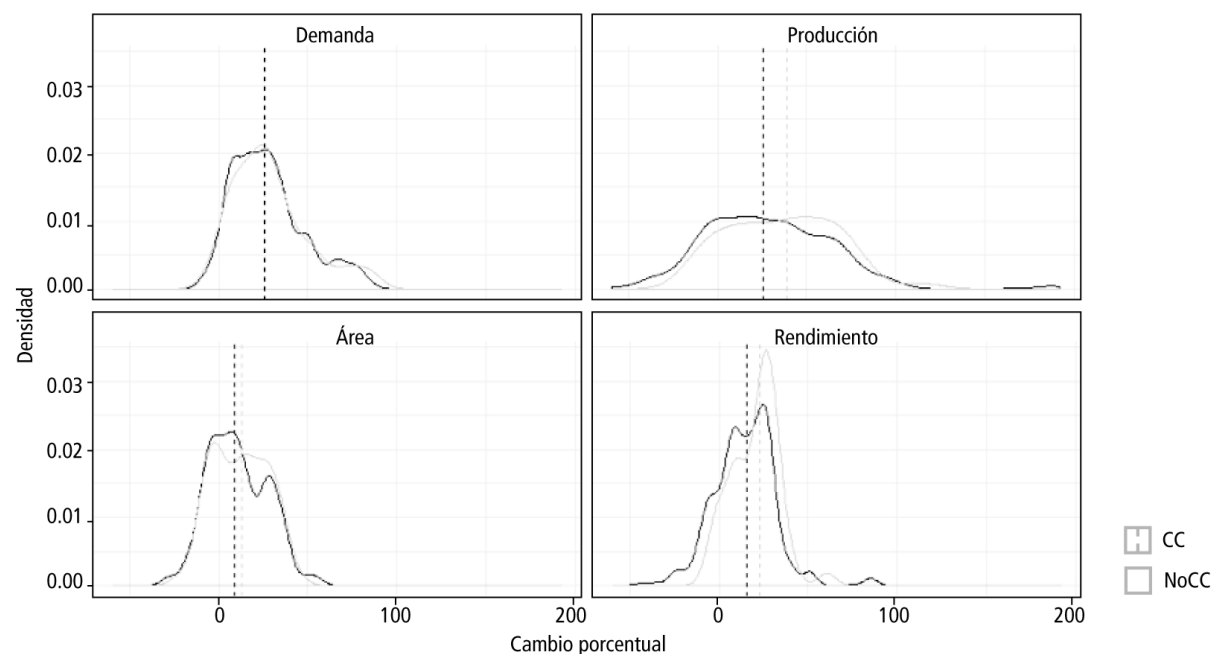

Gráfico 2. Distribución de los cambios porcentuales de la demanda, la producción, el área y los rendimientos en los escenarios construidos

FuENTE: cálculos propios. Notas: la categoría cambio climático (CC) agrupa todos los GCMs analizados. Las distribuciones con cambio climático y sin cambio climático agrupan todos los cultivos y países analizados. El cambio porcentual se calcula con

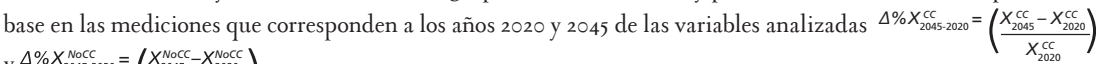
y $\Delta \% X_{2045-2020}^{\text {NocC }}=\left(\frac{X_{2045}^{\text {NoCCC }}-X_{2020}^{\text {NocC }}}{X_{2020}^{\text {NocC }}}\right)$

El número de observaciones por categoría es: demanda con cambio climático $(\mathrm{CC})=$ II25; demanda sin cambio climático $(\mathrm{NoCC})=$ 125; producción con cambio climático $(\mathrm{CC})=873$; producción sin cambio climático $(\mathrm{NoCC})$ = 97; área con cambio climático $(\mathrm{CC})$ = 873; área sin cambio climático $(\mathrm{NoCC})=97$; rendimiento con cambio climático $(\mathrm{CC})=873$; rendimiento sin cambio climático $(\mathrm{NoCC})=97$. 
En cuanto a las áreas cultivadas, los resultados indican que ellas experimentarían reducciones en sus crecimientos. Para el caso del trigo, se proyectan disminuciones en el crecimiento de su área en países como Argentina, Uruguay y Brasil, mientras que en México se presentarían incrementos respecto al escenario en el que no se da el cambio climático. En este último caso, es posible que la competitividad internacional del trigo mexicano disminuya, debido a que las caídas en el crecimiento de los rendimientos estarían acompañadas de incrementos en el área cultivada. Por su parte, el área cultivada de los cultivos de maíz y frijol enfrentaría disminuciones, respectivamente, en países de Centro América, el Caribe, Colombia y Venezuela, al igual que en Brasil y Perú. Por otra parte, en Argentina, las áreas cultivadas de estos cultivos experimentarían incrementos. Finalmente, el área cultivada de arroz presentaría disminuciones significativas en países como Argentina, Chile, Uruguay, Colombia, Venezuela, Brasil, Nicaragua, Guatemala y República Dominicana.

Dados los cambios hasta tanto proyectados de los crecimientos de los rendimientos y las áreas cultivadas, el crecimiento de la producción de los cultivos analizados envolvería diversas transformaciones respecto al escenario carente de cambio climático (NoCC). Específicamente, se encuentra que el crecimiento de la producción de maíz y frijol caería en países como Nicaragua, El Salvador, Guatemala y Honduras, en donde actualmente más de un millón de pequeños agricultores siembra estos cultivos y el consumo es mayor a $25 \mathrm{~kg}$ por persona (Schmidt, Eitzinger, Sonder y Sain, 20I2), y en países de la región Andina como Colombia y Venezuela.

Por otra parte, a nivel regional la producción de arroz sería la que presenta mayores caídas respecto al escenario sin cambio climático (NoCC). Dada la importancia del arroz en ALC, en donde este grano provee más calorías a la dieta de los habitantes que el trigo, el maíz, la yuca y la papa (Degiovanni, Martínez y Motta, 20ro), esta disminución del crecimiento de la producción de arroz podría afectar la seguridad alimentaria regional. Las reducciones en la producción regional estarían impulsadas por las caídas del crecimiento de la producción que se observan en los grandes productores como Brasil, Argentina, Uruguay, Cuba y República Dominicana. Por otra parte, la producción de soya se vería afectada de manera importante en Ecuador y Uruguay, mientras que en los demás productores se experimentaría un impacto neutral o positivo. Finalmente, la producción de trigo tendría efectos especialmente negativos, en particular en grandes productores y comercializadores como Argentina, Brasil y Uruguay. 


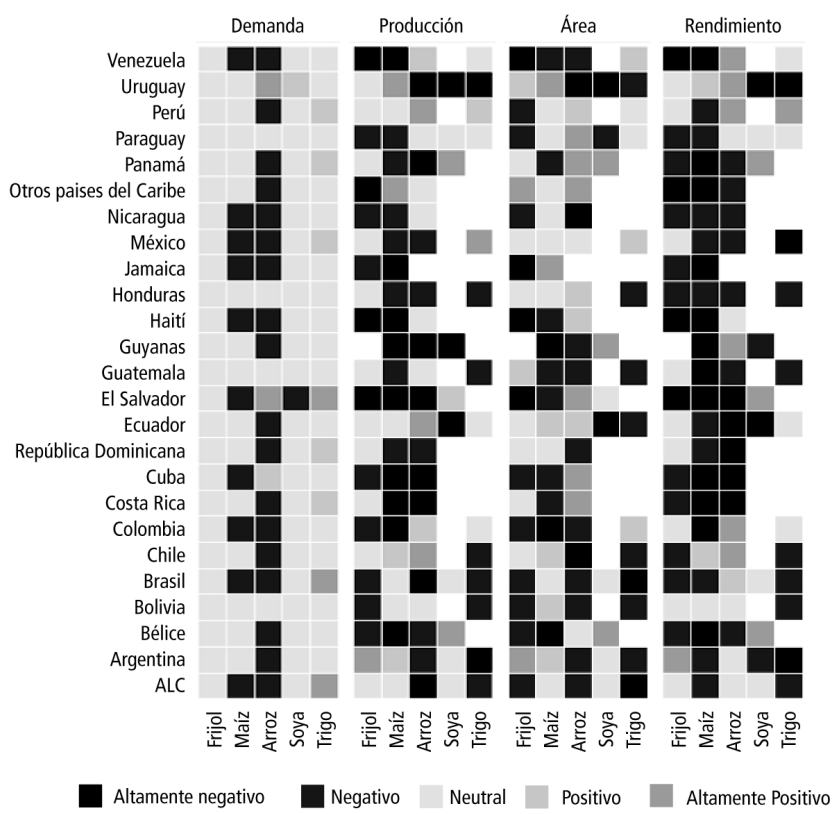

Gráfico 3. Perfil de impacto del cambio climático para la demanda, la producción, el área y el rendimientos por países

Fuente: cálculos propios. Nota: altamente negativo: (- $\infty,-50 \%)$; negativo: (-50\%, -10\%); neutral: (-10\%, 10\%); positivo: (10\%, $50 \%)$; altamente positivo: $(50 \%, \infty)$. Se presentan los resultados de los cambios porcentuales entre las tasas de crecimiento de las variables en el escenario mediano de cambio climático, al igual que en el escenario sin cambio climático .

$\left[\frac{\left(\Delta \% X_{2045-2020}^{\mathrm{CC}}-\Delta \% X_{2045-2020}^{\mathrm{NoCC}}\right)}{\Delta \% X_{2045-2020}^{\mathrm{NoC}}}\right] * 100$

Por el lado de la demanda, los resultados señalan que esta experimentaría un comportamiento heterogéneo entre cultivos, como puede verse en el gráfico 3 . Por una parte, el crecimiento de la demanda de frijol y soya no presentaría grandes cambios respecto al escenario sin cambio climático. Por otra parte, la demanda de maíz, y arroz se vería afectada negativamente en países como Colombia, Venezuela, México y Nicaragua. Finalmente, la demanda de trigo experimentaría un incremento importante, motivado principalmente por el crecimiento que se presentaría en Brasil.

\section{Precios mundiales y comercio internacional}

La evidencia indica que entre 2020 y 2045 los precios de los cultivos analizados aumentarían en términos reales debido a los incrementos de la demanda, que fueron provocados por los crecimientos de los ingresos y la población, así como por los cambios en las preferencias de los consumidores. Asimismo, se observa que el cambio 


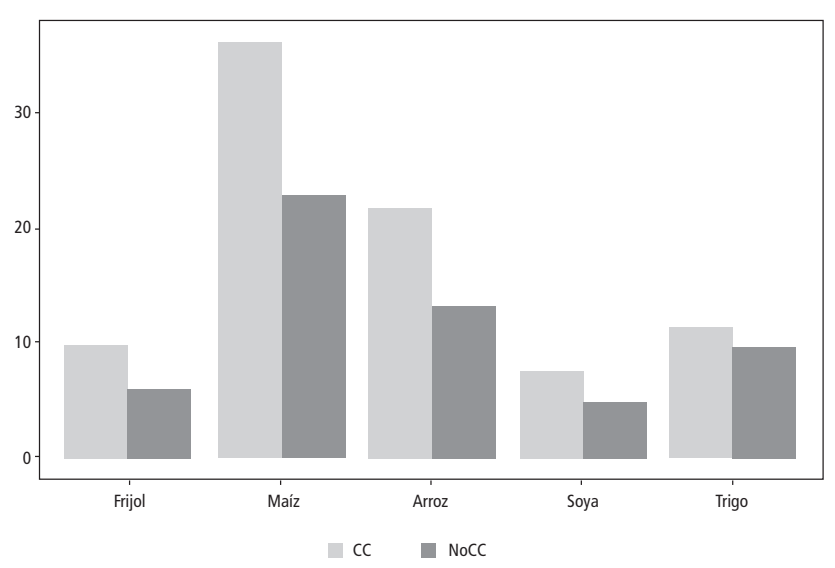

Gráfico 4. Cambios porcentuales de los precios mundiales de los cultivos analizados, con y sin cambio climático entre 2020 y 2045

FuENTE: los autores. Nota: se presentan los resultados para el escenario mediano de cambio climático.

climático generaría presiones adicionales sobre los precios de los cultivos analizados, debido al impacto que tendría en los mercados agrícolas. Los resultados muestran que los precios mundiales del arroz, el frijol, el maíz, la soya y el trigo crecerían, respecto al escenario en el que podría darse el cambio climático, y que corresponde a porcentajes de $66.04 \%, 64.25 \%, 59.54 \%, 59.28 \%$ y $17.32 \%$, respectivamente, como se observa en el gráfico 4 .

En cuanto a los flujos comerciales, los resultados indican que en los escenarios con cambio climático se presentaría un incremento en el crecimiento de las importaciones de trigo, y reducciones en el crecimiento de las exportaciones de frijol, respecto al escenario sin cambios en el clima. Por otra parte, el crecimiento de las importaciones de arroz y maíz disminuiría. Estos resultados sugieren que los impactos del cambio climático sobre los rendimientos también podrían afectar de forma parcial la competitividad relativa de los países de la región que participan en los mercados internacionales de estos cultivos.

\section{Seguridad alimentaria}

Se espera que en América Latina y el Caribe se presenten avances importantes en materia de seguridad alimentaria. Sin embargo, el cambio climático tiene el potencial de frenar parte de los frutos que podrían alcanzarse en esta área. Los resultados señalan que en el escenario mediano de cambio climático, 36 millones de personas estarían en riesgo de padecer hambre, mientras que en un escenario sin cambio climático serían 34 millones de personas. Este incremento en la inseguridad alimentaria sería resultado, 
principalmente, de las disminuciones en la disponibilidad de alimentos en los escenarios con cambio climático, lo que puede constatarse en los gráficos 3 y 6 .

En este punto es importante resaltar dos cosas. Primero, que los cálculos de la población en riesgo de padecer hambre se realizan con base en todos los cultivos y productos presentes en el modelo Impact. Esta es una de las principales limitaciones de este estudio, porque para el caso de los cultivos de frijol, arroz, trigo, soya y maíz, se cuenta con información para nueve GCMs, mientras que para los demás cultivos presentes en el modelo Impact se tiene un promedio de los impactos del cambio

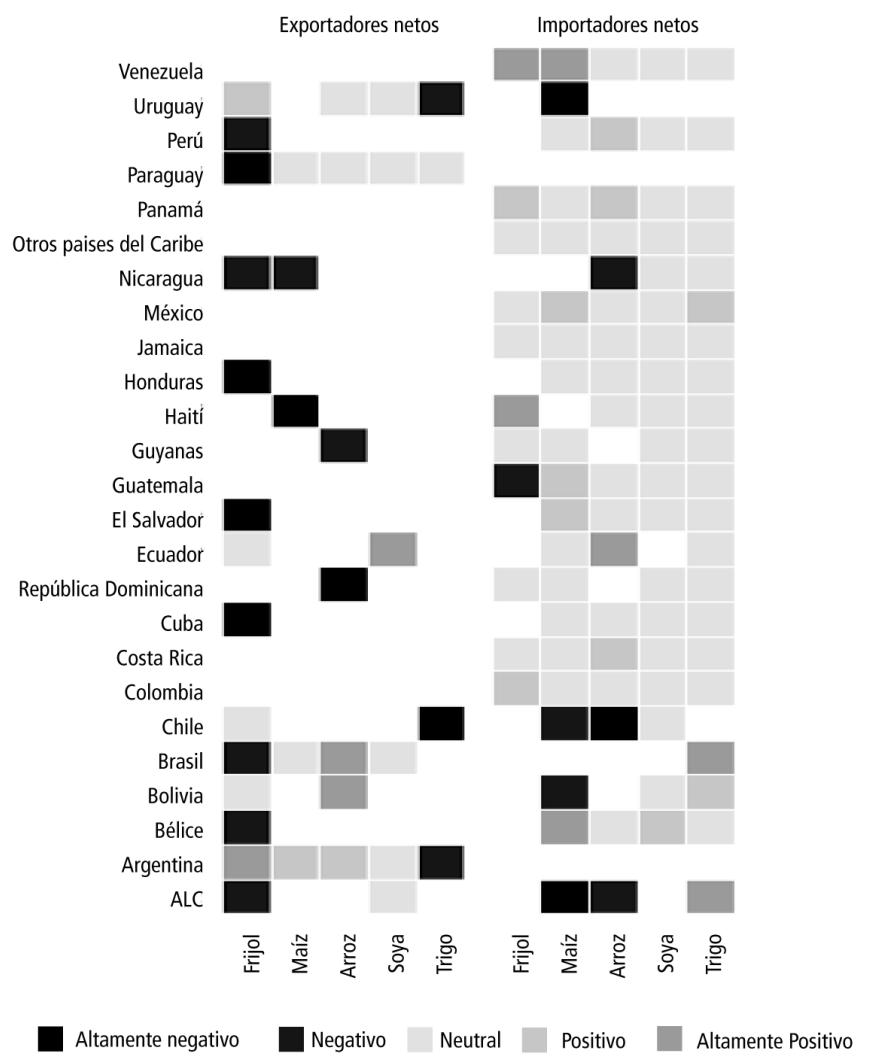

Gráfico 5. Perfil de impacto del cambio climático para las exportaciones netas por países y cultivos (exportadores netos e importadores netos)

FUENTE: los autores. Nota: Se presentan los resultados de los cambios entre el escenario mediano de cambio climático y el escenario sin cambio climático. Cada observación se calcula como el cambio

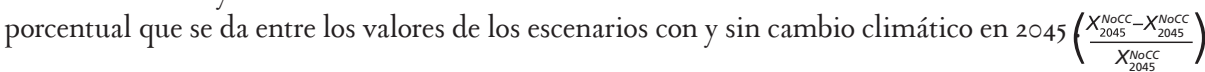

Altamente negativo: (-infinito, $-50 \%)$; negativo: (-50\%, -10 \%); neutral: (-10\%, 10\%); positivo: (10\%, $50 \%)$; altamente positivo: (50\%, infinito). Se presentan los resultados para el escenario mediano de cambio climático. 
climático bajo cuatro GCMs, como puede observarse en la tabla I. En segundo término, el cálculo del porcentaje de la población en riesgo de padecer hambre en el modelo Impact depende principalmente de la disponibilidad de alimentos, y no tiene en cuenta los problemas de acceso económico a los alimentos, los cuales pueden ser un limitante importante en algunos países en desarrollo y desarrollados (FAO, 2003).

Entre los países en donde se presentarían los mayores incrementos en este indicador de inseguridad alimentaria se encuentran Venezuela (17.8\%), Colombia (9.8 \%), Perú (9.4\%), Bolivia (ı \%), y Ecuador (9.5 \%) en la región Andina, y Panamá (II.2 \%), Belice (10.5 \%), República Dominicana (9.3\%), Guatemala (9 \%), Nicaragua (7.3\%), Honduras (7 \%) y Haití (4.3 \%) en Centro América y el Caribe, y México (4.7 \%). Es importante destacar que en el modelo Impact el cálculo del porcentaje de la población en riesgo de padecer hambre tiene una asíntota que se acerca al $4 \%$. Este resultado busca reflejar la idea de que los aumentos de la disponibilidad de alimentos solo pueden mejorar la situación de seguridad alimentaria hasta un punto limitado, y que los efectos positivos que pueden observarse en la reducción de inseguridad alimentara gracias a los incrementos en la disponibilidad de alimentos son decrecientes (Robinson et al., 2015).

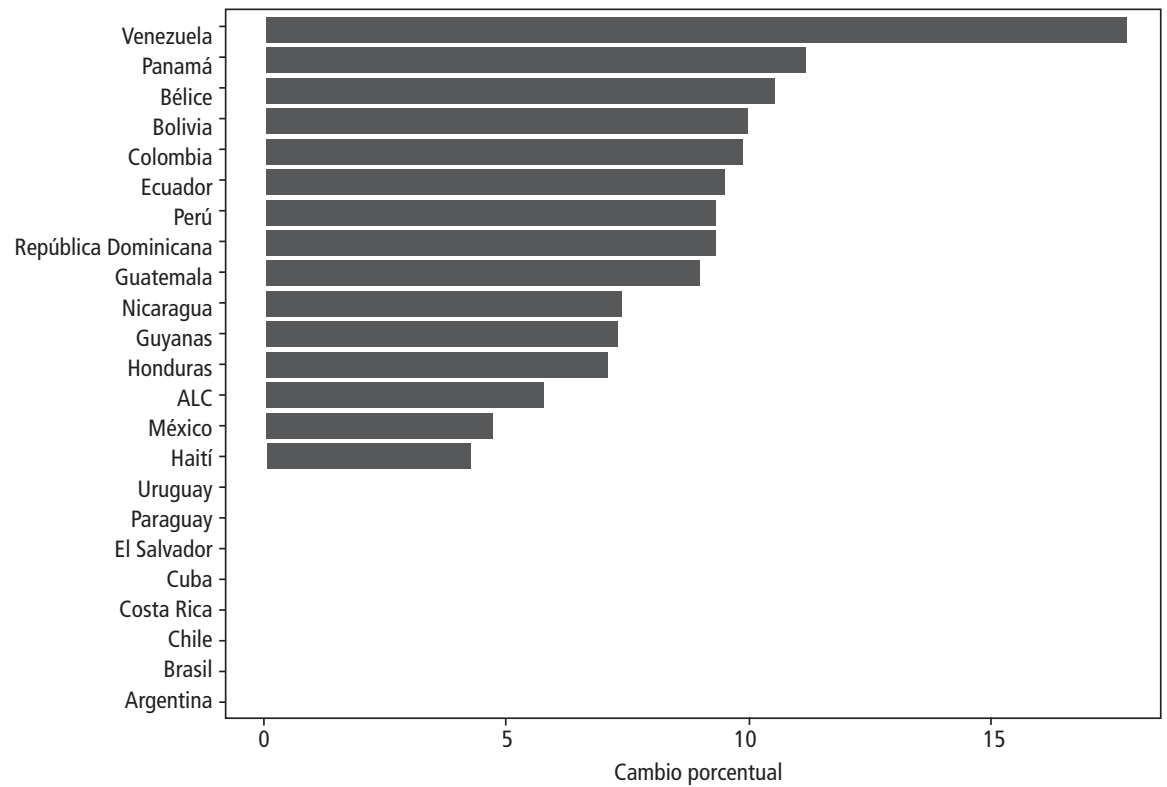

GrÁfico 6. Cambio porcentual en el porcentaje de la población en riesgo de padecer hambre entre escenarios con y sin cambio climático en 2045

Fuente: cálculos propios. Nota: se presentan los resultados de los cambios que se establecen entre el escenario mediano de cambio climático y el escenario sin cambio climático. Cada observación se calcula como el cambio porcentual que se origina entre los valores de los escenarios con y sin cambio climático en 2045; cambio porcentual $=\left(\frac{X_{2045}^{\mathrm{cC}}-X_{2045}^{\mathrm{NocC}}}{X_{2045}^{\mathrm{NoCC}}}\right)$ 


\section{Discusiones}

Los resultados obtenidos en este estudio muestran que el cambio climático tiene el potencial de afectar los rendimientos, el área cultivada y la producción agrícola, y que dichos impactos son específicos para cada cultivo y país. A pesar de la diversidad de los efectos del cambio climático, es notable que su impacto mediano sería negativo en los rendimientos, el área cultivada y la producción de frijol, soya, arroz, trigo y maíz. A diferencia de lo que puede observarse a partir de los resultados aquí encontrados, con base en las investigaciones de Nelson et al. (20I4) es posible concluir que el impacto negativo del cambio climático sobre los rendimientos provocaría que los agricultores, además de intensificar el uso de prácticas de manejo, incrementaran el área cultivada. Las diferencias que se observan entre nuestros resultados pueden deberse a varias razones. Primero, en el trabajo mencionado se utilizan nueve modelos económicos, mientras que aquí solamente se utiliza uno. Segundo, en dicho estudio se presentan resultados a nivel mundial, mientras que aquí solo se analizan los países de ALC. Tercero, en dicho estudio se analizan los cultivos de trigo, cereales secundarios, arroz y semillas oleaginosas, mientras que aquí se analizan el frijol, el maíz, la soya, el arroz y el trigo.

La respuesta endógena del área es importante ya que determina, junto con los cambios en los rendimientos, el impacto que el cambio climático tiene en la producción. Los resultados aquí presentados señalan que el crecimiento del área cultivada regional de frijol, arroz y trigo, sería inferior en los escenarios con cambio climático (CC), mientras que los crecimientos de las áreas de maíz y soya presentarían un patrón similar al que fue exhibido en el escenario que no presentaba cambios en el clima (NoCC). Como resultado de los cambios que se han observado en los rendimientos y las áreas cultivadas hasta ahora mencionados, los crecimientos de las producciones presentarían alteraciones importantes respecto al escenario en el que no se daban cambios en el clima (NoCC). Dichos resultados son notables especialmente en los casos de los cultivos de arroz y trigo, aunque los cambios que se desencadenaron en el crecimiento de la producción de arroz serían motivados principalmente por las caídas en el crecimiento del área cultivada, mientras que en el caso del trigo serían la consecuencia de las reducciones en el crecimiento de los rendimientos y de las áreas.

En contraste con lo que muestran otras variables, se observan similitudes entre el crecimiento mediano de la demanda de los cinco cultivos en los escenarios en los que se da el cambio climático, y los que no lo evidencian. Además, aunque se presentan 
diferencias entre los patrones de crecimiento que correspondían a la demanda de los cultivos, el crecimiento que se observa entre los países es relativamente homogéneo para cada cultivo. Estos resultados sugieren un alto grado de inelasticidad de la demanda por los cultivos que han sido analizados ante los incrementos en los precios de los cultivos; cambios que, por su parte, han sido provocados por el impacto del cambio climático en los rendimientos. Estos resultados son consistentes con los hallazgos que han surgido del trabajo de Wiebe et al. (2015), en donde se comparan los resultados de cinco modelos económicos bajo un conjunto de escenarios socioeconómicos y RCPs.

Como fue mencionado anteriormente, las presiones del cambio climático sobre la producción agrícola generarían incrementos en el crecimiento de los precios del arroz, el trigo, el maíz, el frijol y el arroz. Estos resultados son también consistentes con los encontrados en el trabajo de Rosegrant et al. (20r4) en donde, mediante una metodología similar a la empleada en este estudio, se encuentra que los precios del maíz, el trigo y el arroz, presentarían incrementos superiores al 60 \% entre 2010 y 2050 en escenarios caracterizados por el cambio climático.

\section{Conclusiones}

De entrada vale la pena considerar que la evaluación de los impactos del cambio climático es un proceso que tiene distintos tipos de incertidumbre que se asocian con los materiales y los métodos que son empleados en las distintas fases de la modelación. Los resultados aquí presentados deben leerse como posibles escenarios que podrían presentarse, dados los supuestos planteados en las distintas etapas de la modelación, tales como corresponde a los crecimientos poblacionales y económicos que atañen a la productividad agrícola y los cambios que se dan en el clima. A pesar de la incertidumbre que se presenta en este tipo de trabajos, existe un conjunto consistente de evidencia que señala que el cambio climático puede tener un impacto significativo sobre los mercados agrícolas, así como sobre la seguridad alimentaria -para lo que vale la pena revisar los trabajos de Wiebe et al. (2015), Rosegrant et al. (20I4), y Nelson et al. (20I4)-. Los resultados aquí presentados constituyen una contribución significativa a la literatura que versa sobre el tema, al evaluar los impactos socioeconómicos del cambio climático a nivel de país en ALC. Haciendo esta desagregación a nivel de país se busca ilustrar la heterogeneidad que caracteriza 
los impactos del cambio climático en los mercados agrícolas del frijol, el arroz, la soya, el trigo y el maíz, así como en la seguridad alimentaria.

Así pues, las conclusiones más importantes de este artículo son tres. Primero, que el cambio climático afectaría negativamente los crecimientos de los rendimientos, las áreas cultivadas, y las producciones de los cultivos analizados. Segundo, que este fenómeno natural tiene el potencial de limitar los avances que en materia de seguridad alimentaria se alcanzarían en su ausencia. En otras palabras, debido a las disminuciones en la disponibilidad de alimentos, dos millones de personas adicionales estarían en riesgo de padecer hambre, en comparación con un escenario que no tuviera tales cambios en el clima.

En tercera medida, la evidencia encontrada muestra que los impactos del cambio climático serán específicos por cultivos y países. Resulta notable que el crecimiento de la producción de maíz y frijol caería principalmente en Nicaragua, El Salvador, Guatemala, Honduras, Colombia, Venezuela y Brasil. Por otra parte, a nivel regional la producción de arroz es la que presentaría mayores caídas con respecto al escenario en que no se da el cambio climático (NoCC). Estas reducciones de la producción regional estarían impulsadas por las caídas en el crecimiento de la producción en Brasil, Argentina, Uruguay, Cuba y República Dominicana. En cuanto a la producción de soya, esta se vería afectada de manera importante en Ecuador y Uruguay, mientras que en los demás productores se experimentaría un impacto neutral o positivo. Finalmente, la producción de trigo sería afectada negativamente, especialmente en grandes productores y comercializadores como Argentina, Brasil y Uruguay.

Debido a la heterogeneidad de los cambios en el clima, que corresponden a patrones de lluvia e incrementos de las temperaturas, las medidas que deberían tomarse en la región para adaptarse a sus efectos variarían entre países y cultivos. Entre las estrategias sugeridas se encuentran el desarrollo de nuevas tecnologías y conocimientos, así como la transferencia de tecnologías ya existentes. Por ejemplo, el desarrollo de variedades cuyas características permiten la resistencia a la sequía y a las altas temperaturas, al igual que la implementación de programas de proyección climática que ofrezcan información alusiva a los posibles cambios que pueden haber en los patrones de lluvias y temperaturas en el corto y mediano plazo. Lo mismo corresponde a la promoción de programas sociales que incentiven la inversión en proyectos de distritos de riego, cosecha de agua, así como en la producción y comercialización de semillas certificadas. Por medio de este tipo de intervenciones se podría promover la resiliencia de los pequeños y grandes agricultores ante los cambios 
del clima, lo cual permitiría garantizar la sostenibilidad de los mercados agrícolas de la región, y su competitividad en el escenario internacional.

Finalmente, con el fin de manejar mejor la incertidumbre que corresponde a este tipo de evaluaciones, en futuras investigaciones se debería incluir en el análisis un mayor número de cultivos, así como de modelos climáticos, de cultivos y económicos. Por lo mismo, valdría la pena tener en cuenta un mayor número de trayectorias plausibles del crecimiento poblacional y económico. De este modo se podrían obtener resultados más robustos, que contemplaran los posibles impactos del cambio climático en los mercados agrícolas, así como la seguridad alimentaria de los países latinoamericanos.

\section{Agradecimientos}

Agradecemos el apoyo brindado por los programas del Cgiar en Cambio Climático, Agricultura y Seguridad Alimentaria (Ccafs), y los correspondientes a Políticas, Instituciones y Mercados (PIM). Los escenarios presentados en este estudio fueron construidos con base en el trabajo que fue desarrollado con el apoyo de los proyectos Global Futures \&- Strategic Foresight (GFSF), y Climate change vulnerability in the agricultural sector in Latin America and the Caribbean, este último financiado por el Banco Interamericano de Desarrollo (BID). Esta investigación también fue posible gracias al apoyo del Centro Internacional de Agricultura Tropical (CIAT), y del International Food Policy Research Institute (Ifpri). Asimismo, este artículo es una extensión del proyecto Climate change vulnerability in the agricultural sector in Latin America and the Caribbean.

\section{Bibliografía}

Darwin, R. F. (1998). Farm: a Global Framework for Integrated Land Use / Cover Modeling. Working Papers in Ecological Economics (9802). Recuperado de http:// digitalcollections.anu.edu.au/handle/1885/40914

Degiovanni, V., Martinez, C. y Motta, F. (2010). Producción eco-eficiente del arroz en América Latina. Centro Internacional de Agricultura Tropical (CIAT). Recuperado de http://ciat-library.ciat.cgiar.org/Articulos_Ciat/2010_Degiovanni-Produccion_ eco-eficiente_del_arroz.pdf 
Evenson, R., Pray, C. y Rosegrant, M. (1999). Agricultural Research and Productivity Growth in India (Ifpri Research Report No. I09). International Food Policy Research Institute.

FAO (2003). Trade Reforms and Food Security Conceptualizing the Linkages. Recuperado de http://www.fao.org/3/a-y467ie.pdf

FAO, liasa, Isric, ISS-CAS y JRC. (20I2). Harmonized World Soil Database (version I.2).

Fischer, G., Shah, M., Tubiello, F. N. y Velhuizen, H. van. (2005). Socio-Economic and Climate Change Impacts on Agriculture: an Integrated Assessment, 19902080. Philosophical Transactions of the Royal Society of London B: Biological Sciences, 360(I463), 2067-2083. https://doi.org/I0.I098/rstb.2005.I744

Fujimori, S., Masui, T. y Matsuoka, Y. (20I2). AIM/CGE [Basic] Manual. Discussion Paper Series Center for Social and Environmental Systems Research, NIES. Recuperado de http://www.nies.go.jp/social/dp/pdf/20I2-or.pdf

Gourdji, S. M., Sibley, A. M. y Lobell, D. B. (20I3). Global Crop Exposure to Critical High Temperatures in the Reproductive Period: Historical Trends and Future Projections. Environmental Research Letters, 8. Recuperado de http://iopscience.iop. org/article/ıo.1088/1748-9326/8/2/02404I/meta

Gourdji, S., Mesa-Diez, J., Obando-Bonilla, D., Moreno-Cadena, P., NavarroRacines, M., Fisher, C., Prager, S. D., y Ramirez-Villegas, J. (20I6). Near-term impacts of climate change on yields of five major crops across Latin America and the Caribbean (pp. I-50). Palmira, Colombia: Centro Internacional de Agricultura Tropical (CIAT).

Havlík, P., Valin, H., Herrero, M., Obersteiner, M., Schmid, E., Rufino, M. C., Monsier, A., Thornton, P. K., Böttcher, H., Conant, R. T., Frank, S., Fritz, S., Fuss, S., Kraxner, F. y Notenbaert, A. (20I4). Climate Change Mitigation through Livestock System Transitions. Proceedings of the National Academy of Sciences, III(IO), 3709-3714. https://doi.org/10.1073/pnas.I308044III

IPCC Working Group. (20I3). The Physical Science Basis. Recuperado de http://www. climatechange2013.org/

Jarvis, A., Ramirez, J., Herrera, B. V. y Navarro, C. (20I2). Is Cassava the Answer to African Climate Change Adaptation? Tropical Plant Biology, 5(I), 9-29. https://doi. org/I0.1007/SI2042-0I2-9096-7

Jones, J., Hoogenboom, G., Porter, C., Boote, K., Batchelor, W., Hunt, L., Wilkens, P., Singh, U., Gijhsman, A. y Ritchie, J. (2003). The Dssat Cropping System Model. Modelling Cropping Systems: Science, Software and Applications, I8(3-4), 235-265. https:// doi.org/I0.I0I6/SII6I-030I(02)00107-7 
Lobell, D. B., Banziger, M., Magorokosho, C. y Vivek, B. (20II). Nonlinear Heat Effects on African Maize as Evidenced by Historical Yield Trials. Nature Climate Change, $I(\mathrm{I}), 42-45$. https://doi.org/10.1038/nclimatero43

Meadu, V., Coche, I., Vermeulen, S. y Engelund Friss, A. (2015). The Paris Climate

Agreement: What it Means for Food and Farming (CCAFS Info Notes).

Recuperado de https://cgspace.cgiar.org/rest/bitstreams/64038/retrieve

Nelson, G., Valin, H., Sands, R. D., Havlík, P., Ahammad, H., Deryng, D., Elliott,

J., Shinichiro, F., Hasegawa, T., Edwina, H., Page, K., Von Lampe, M., Lotze-

Campen, H., Mazon d'Cros, D., Van Mejil, H., Van Der Mensbrugghe, D.,

Müller, C., Propp, A., Robertson, R., Robinson, S., Schmid, E., Schitz, C.,

Tabeau, A. y Willenbockel, D. (20I4). Climate Change Effects on Agriculture:

Economic Responses to Biophysical Shocks. Proceedings of the National Academy of

Sciences, ${ }_{1 I I}(9)$, 3274-3279. https://doi.org/10.1073/pnas.1222465110

O’Neill, B., Kriegler, E., Riahi, K., Ebi, K. L., Hallegate, S., Carter, T., Mathur,

R. y van Vuuren, D. P. (2014). A New Scenario Framework for Climate Change

Research: the Concept of Shared Socioeconomic Pathways. Climatic Change, I22(3),

387-400. https://doi.org/10.1007/s10584-013-0905-2

PIK Landuse Group (20II). MAgPIE Mathematical Description. Recuperado de https://

www.pik-potsdam.de/research/sustainable-solutions/research/landuse-group/ magpie-mathematical-description

Portman, F., Siebert, S. y Doll, S. (2010). Global Monthly Irrigated and Rainfed Crop Areas Around the Year 2000: A New High-Resolution Data Set for Agricultural and Hydrological Modeling. Global BiogeochemicalCycles, 24(I). https://doi.

org/10.1029/2008GB०0 3435

Robinson, S., Mason-D’Croz, D., Islam, S., Sulser, T., Gueneau, A., Pitois, G. y Rosegrant, M. W. (2015). The International Model for Policy Analysis of Agricultural Commodities and Trade (IMPACT): Model description for version 3 (Ifpri Discussion Paper). Washington, D.C: International Food Policy Research Institute.

Recuperado de http://ebrary.ifpri.org/cdm/ref/collection/p15738coll2/id/ı29825

Rosegrant, M. W., Koo, J., Cenacchi, N., Ringler, C., Robertson, R., Fisher, M., Cox, C., Garrett, K., Nicostrato, P. y Sabbagh, P. (2014). Food Security in a World of Natural Resource Scarcity: the role of Agricultural Technologies. International Food Policy Research Institute. Recuperado de http://dx.doi.org/I0.2499/9780896298477 Schmidt, A., Eitzinger, A., Sonder, K. y Sain, G. (2012). Tortillas on the Roaster. Central American Maize-Bean Systems and the Changing Climate. Recuperado de http://www. crs.org/sites/default/files/tools-research/tortillas-on-the-roaster-full-report_o.pdf 
Vaghefi, N., Nasir Shamsudin, M., Radam, A. y Rahim, K. (2013). Modelling the Impact of Climate Change on Rice Production: An Overview. Journal of Applied Sciences, I3(24). https://doi.org/http://dx.doi.org/10.3923/jas.2013.5649.5660

Van der Mensbrugghe, D. (2010). The Environmental Impact and Sustainability Applied General Equilibrium (Envisage) Model. The World Bank. Recuperado de http:// go.worldbank.org/DURRYWVEZo

Wiebe, K., Lotze-Campen, H., Sands, R., Tabeau, A., Mensbrugghe, D. van der Biewald, A., Bodirsky, B., Islam, S., Kavallari, A. y Willenbockel, D. (2015).

Climate Change Impacts on Agriculture in 2050 Under a Range of Plausible Socioeconomic and Emissions Scenarios. Environmental Research Letters, io (8), 85010. You, L., Wood-Sichra, U., Fritz, S., Guo, Z., See, L. y Koo, J. (2014). Spatial Production Allocation Model (SPAM). Recuperado de http://mapspam.info/ 